Check for updates

Cite this: RSC Adv., 2019, 9, 11818

\title{
Tunable gap in stable arsenene nanoribbons opens the door to electronic applications $\uparrow$
}

\begin{abstract}
A. García-Fuente, (D) *a J. Carrete, (D) ${ }^{\text {b } A . ~ V e g a c ~ a n d ~ L . ~ J . ~ G a l l e g o ~(D) ~ d ~}$
Arsenic has been predicted to present significantly more diverse 2D phases than other elemental compounds like graphene. While practical applications must be based on finite arsenene samples, like nanoribbons, theory has so far focused on the infinite sheet. Our ab initio simulations show the clear contrast between the properties of arsenene nanoribbons and those of the monolayer, ranging from phase stability to electronic structure. We include nanoribbons derived from the buckled, puckered and square/octagon structures of bulk arsenene. The flexibility afforded by different parent structures, widths and edge passivations leads to a rich variety of semiconducting structures with tunable gaps.
\end{abstract}

Received 5th February 2019

Accepted 9th April 2019

DOI: 10.1039/c9ra00975b

rsc.li/rsc-advances of them are semiconductors with buckled (b), puckered (p), or square/octagon (s/o) structures, and fundamental band gaps ranging from 3 to $0.15 \mathrm{eV}$, rendering them potentially suitable for diverse applications in 2D electronics.

Despite these encouraging results, the topic is far from closed. Some of us have recently shown ${ }^{14}$ that, due to phonon contributions not included in previous studies, the relative stability of the b, p and s/o proposed phases of arsenene ${ }^{13,15,16}$ is both temperature dependent and clearly at odds with existing predictions. This finding is crucial since accurate determination of the stable geometry of a nanostructure, in general, is a prerequisite to precisely establishing its physico-chemical properties. Similarly, those pioneering studies restricted themselves to the infinite monolayer of each 2D structure, but real devices require specific nanostructures with particular geometries. Although some theoretical studies on arsenene and antimonene nanoribbons in the buckled form have been performed, ${ }^{17}$ most efforts in this domain have concentrated on phosphorene nanoribbons; for instance, Zhang et al. ${ }^{18}$ have studied their electronic and transport properties for different widths and edge configurations, predicting them to have very high figures of merit at room temperature. Motivated by the structural diversity of arsenenes, here we report an ab initio density functional theoretic (DFT) study of the structures, stability and electronic properties of arsenene nanoribbons (AsNRs) including both phonons and edge effects. We show that by conceptually cutting the three proposed stable structures of 2D arsenene indicated above ( $\mathrm{b}, \mathrm{p}$ and $\mathrm{s} / \mathrm{o}$ ) along different directions we obtain a large variety of nanostructures with electronic properties of interest. In particular, the band gaps of AsNRs can be tuned within a wide range by selecting the 2D parent structure, the cutting direction, and the ribbon width. Moreover, the relative stability of the b, p, and s/o AsNRs does not simply follow that of the bulk $2 \mathrm{D}$ phases. Hence, although only supported, multilayer AsNRs have already been
${ }^{a}$ Departamento de Física, Universidad de Oviedo, E-33007 Oviedo, Spain. E-mail: garciaamador@uniovi.es

${ }^{b}$ Institute of Materials Chemistry, TU Wien, A-1060 Vienna, Austria

'Departamento de Física Teórica, Atómica y Óptica, Universidad de Valladolid, E47011 Valladolid, Spain

${ }^{d}$ Departamento de Física de la Materia Condensada, Facultad de Física, Universidad de Santiago de Compostela, E-15782 Santiago de Compostela, Spain

$\dagger$ Electronic supplementary information (ESI) available. See DOI: 10.1039/c9ra00975b 
synthesized, ${ }^{19}$ our results point to the interest of analyzing the potential uses of these nanostructures.

Our calculations were performed using $\mathrm{VASP}^{20,21}$ and the Phonopy package. ${ }^{22}$ Details of the method can be found in the ESI. $\dagger$

\section{Results and discussion}

Our starting point are the geometric and electronic structures of the infinitely extended b, p and s/o arsenene sheets, as obtained by DFT calculations. In ref. 14 some of us performed optimizations of these structures within the Perdew-Burke-Ernzerhof (PBE) form of the GGA, ${ }^{23}$ and the results are reproduced in ESI Fig. $1 . \dagger$ To complete the information, we now computed their electronic band structures using both PBE and the hybrid functional HSE06. ${ }^{24-27}$ Our results, shown in ESI Fig. $2, \dagger$ are in good agreement with those reported by Ciraci and coworkers. $^{13,28}$ The effect of the HSE correction essentially amounts to a rigid displacement of all bands away from the center of the gap. Therefore the fundamental band gaps are substantially increased, as can be seen in ESI Table $1 . \dagger$

By conceptually cutting nanometer-scale-width strips out of the $\mathrm{b}, \mathrm{p}$ and $\mathrm{s} / \mathrm{o}$ arsenene structures along the directions indicated in ESI Fig. $1, \dagger$ we obtained nanoribbons that share their basic geometries with their $2 \mathrm{D}$ precursors and have edges of different shapes. Specifically, we obtained zigzag and armchair buckled arsenene nanoribbons (zz-b-AsNRs and ac-b-AsNRs), zigzag and armchair puckered arsenene nanoribbons (zz-pAsNRs and ac-p-AsNRs), and "toothy" and armchair squareoctagon arsenene nanoribbons (t-s/o-AsNRs and ac-s/oAsNRs). We considered ribbon widths ranging from 5 to $30 \AA$, both with free edge atoms and with hydrogen passivation along the edges - a common practice to stabilize nanoribbons. Hydrogen-passivated arsenene nanoribbons will be denoted as H-zz-b-AsNRs and so on and so forth. Fig. 1 shows the relaxed structures of an illustrative sample of AsNRs with similar widths of around $15 \AA$, and Fig. 2 shows their bond-length distributions. The upper panels of Fig. 3 shows the cohesive energies per atom of all the nanoribbons investigated in this paper. Those are calculated as

$$
E_{\mathrm{c}}=\frac{n_{\mathrm{As}} E(\mathrm{As})+\frac{n_{\mathrm{H}}}{2} E\left(\mathrm{H}_{2}\right)-E(\mathrm{AsNR})}{n_{\mathrm{As}}},
$$

where $E(\mathrm{As}), E\left(\mathrm{H}_{2}\right)$ and $E(\mathrm{AsNR})$ are the total DFT energies of an As atom, an $\mathrm{H}_{2}$ molecule and the AsNR unit cell, and $n_{\mathrm{As}}$ and $n_{\mathrm{H}}$ are the number of As and $\mathrm{H}$ atoms in the unit cell. In general, nanoribbons without edge passivation undergo edge reconstructions, an effect that disappears when the edges are passivated by hydrogen atoms. In fact, hydrogen passivation generally stabilizes the nanoribbons, introducing an energetic ordering equal to that of the 2D precursors (Fig. 3, upper panels). The only exceptions occur for the two narrowest t-s/oAsNRs, which are more stable than the corresponding $\mathrm{H}-\mathrm{t}-\mathrm{s} / \mathrm{o}-$ AsNRs. This particularly interesting class of AsNRs experiments a dramatic reconstruction upon relaxation, adopting a structure that can be described as a sequence of nanowires

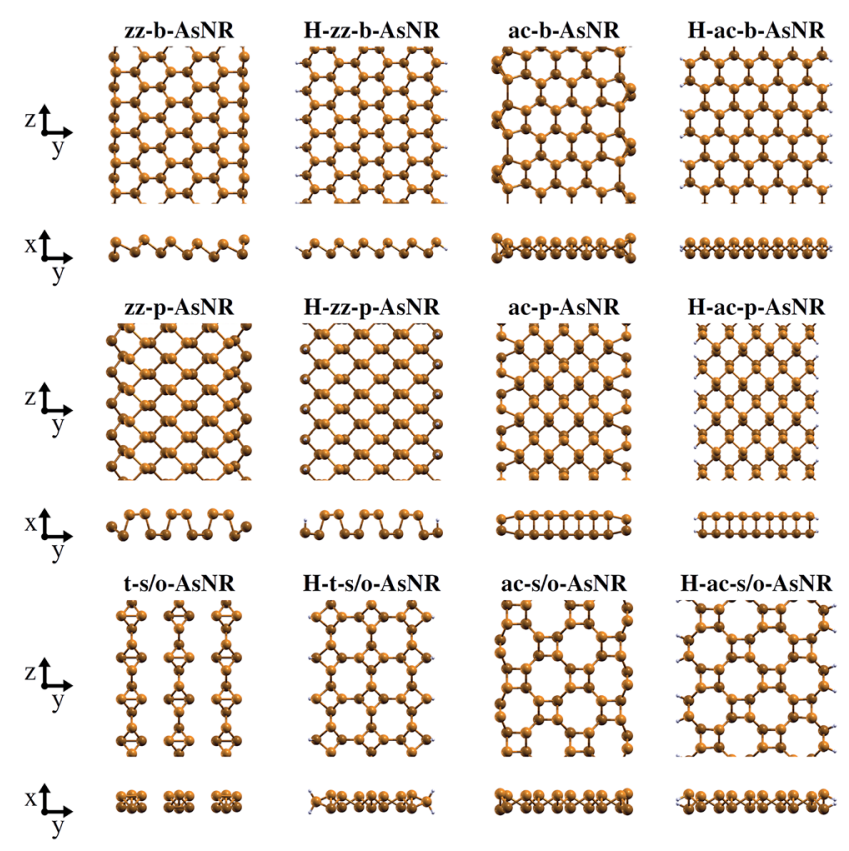

Fig. 1 Top and front views of the relaxed structures of illustrative nonpassivated and hydrogen-passivated AsNRs.

with little interaction between them. This can explain why their cohesive energy per atom is largely independent of width. We note that these nanowires are formed by apical-bounded rhombohedral units in which, in addition to the rhomboidforming As-As bonds, there is a transannular As-As bond. Thus, each As atom of each nanowire is coordinated to three neighboring atoms of the same wire, saturating their valence electrons. In Fig. 4 we show the electronic charge density difference between a t-s/o-AsNR and the array of parallel, noninteracting As nanowires that can be taken as its building blocks. The results point to an electronic bond being formed
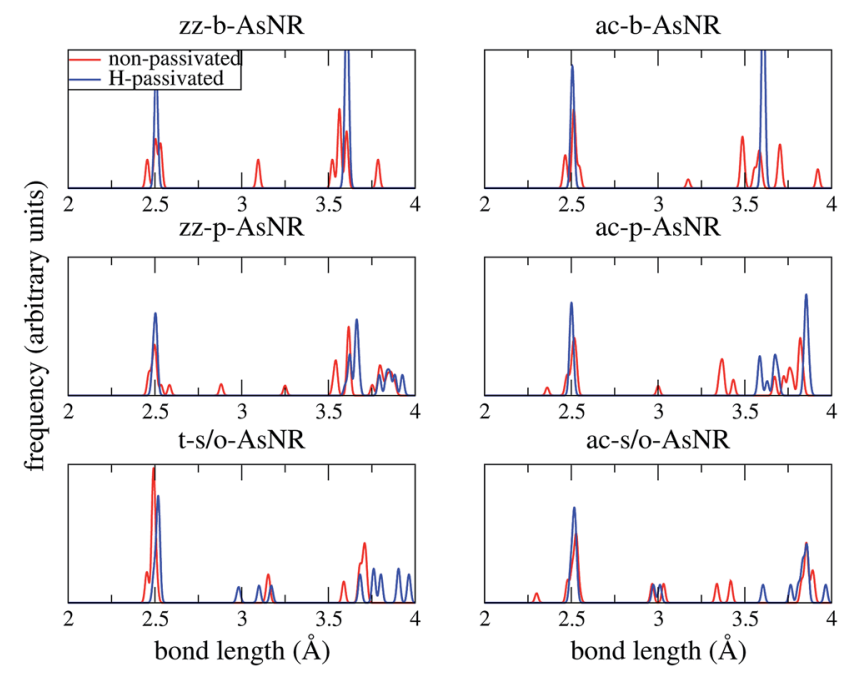

Fig. 2 Bond-length distributions of the passivated and non-passivated AsNRs shown in Fig. 1. 

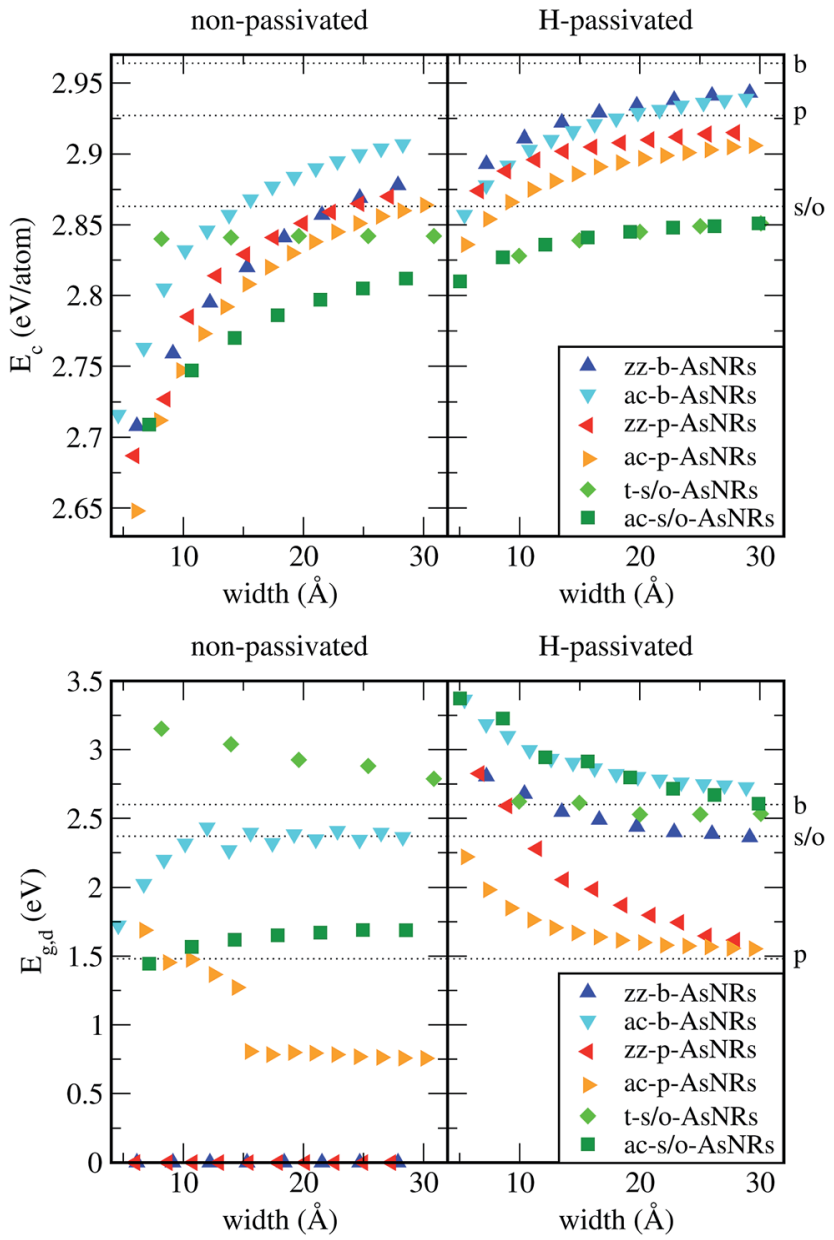

Fig. 3 Upper panels: cohesive energies per atom, $E_{c}$, of the nonpassivated and hydrogen-passivated AsNRs as functions of their widths; the dashed lines correspond to the cohesive energies for the periodic, infinitely extended, $\mathrm{p}, \mathrm{b}$ and $\mathrm{s} / \mathrm{o}$ arsenene sheets. Lower panels: direct band gaps of the non-passivated and hydrogenpassivated AsNRs as functions of their widths, calculated with HSE; the dashed lines correspond to the direct band gaps for the periodic, infinitely extended, $\mathrm{p}, \mathrm{b}$ and $\mathrm{s} / \mathrm{o}$ arsenene sheets.

between the closest As atoms belonging to neighboring nanowires.

In order to see if the peculiar reconstruction of the t-s/oAsNRs disappears when the ribbon width is larger than a critical value, we performed calculations on a periodic 2D arrangement of nanowires such as would be obtained if the whole infinite sheet experienced this "lateral dimerization". The results show that the structure formed by nanowires is also stable in the infinite case, with a cohesive energy of $2.844 \mathrm{eV}$ per atom that is not far from the value we had obtained for the nanoribbons (2.842 eV per atom). Note that the infinite sheet, with $2.86 \mathrm{eV}$ per atom, is more stable also in this case, but both could very well be observed given that the difference is lower than the room-temperature value of $k_{\mathrm{B}} T$, where $k_{\mathrm{B}}$ is the Boltzmann constant. Which structure actually appears under specific conditions will depend on the details of the attraction basin of

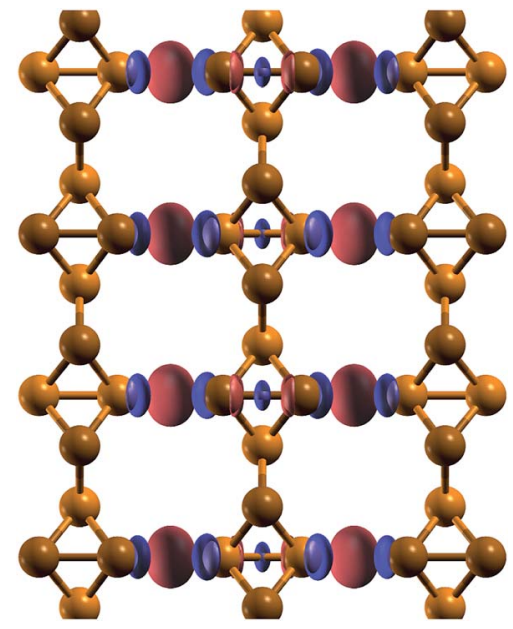

Fig. 4 Electronic charge density difference between a t-s/o-AsNR and isolated t-s/o-nanowires. Positive values of the density difference are shown in red, and negative values are shown in blue.

each minimum. In conclusion, no crossover width exists for the phenomenon.

We calculated the band structures of all the AsNRs considered in this paper using both PBE and HSE, and the upper panels of Fig. 5 show the results for the nanoribbons represented in Fig. 1. In the lower panels of Fig. 3 we display the corresponding energy gaps as a function of their widths, obtained using the HSE functional (PBE results are given in the $\mathrm{ESI} \dagger$ ). Due to the edge reconstruction, the variation of the gap in non-passivated AsNRs is rather irregular. The most striking examples are provided by zz-b-AsNRs and zz-p-AsNRs, which are metallic rather than semiconducting (see Fig. 5, upper panels). The exceptions are, again, the t-s/o-AsNRs, whose particular geometry leads to a regular decrease of the band gap with width. On the other hand, the electronic behavior of the hydrogenpassivated AsNRs is much more regular (upper panels of Fig. 5 and lower right panel of Fig. 3), as a consequence of their general stability. Those nanoribbons are all semiconductors, with band gaps that increase when the widths decrease as a manifestation of confinement effects. The largest band gaps occur for $\mathrm{H}$-ac-s/o-AsNRs and $\mathrm{H}$-ac-b-AsNRs and the smaller ones for $\mathrm{H}$-ac-p-AsNRs. Widths around $30 \AA$ are enough to recover the band gap of the infinite $2 \mathrm{D}$ precursors. Additional information on the electronic properties of the AsNRs (specifically, the room temperature carrier densities and mobilities, and the electronic transmittances) is given in the ESI, $\dagger$ corroborating the electronic character already inferred from the energy band structures shown in Fig. 5 .

It should be noted that some of the results presented above for zz-p-AsNRs and ac-p-AsNRs are qualitatively similar to those obtained by Zhang et al. ${ }^{18}$ for zigzag and armchair puckered (or black) phosphorene nanoribbons (zz-p-PNRs and ac-p-PNRs) using the PBE form of the GGA. In particular, they found that zz-p-PNRs are metallic, while ac-p-PNRs are semiconducting. However, after hydrogen passivation of the edge atoms zz-pPNRs become semiconducting, while ac-p-PNRs exhibit larger 


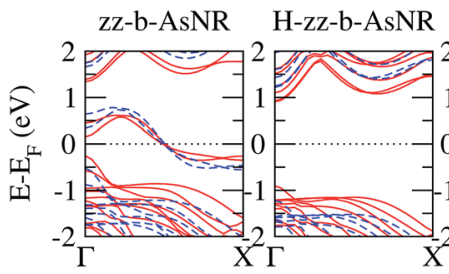

ac-b-AsNR

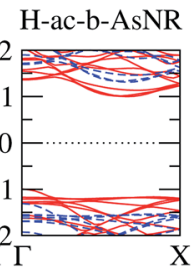

zz-p-AsNR H-zz-p-AsNR
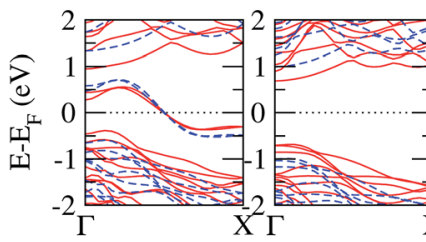

ac-p-AsNR

H-ac-p-AsNR

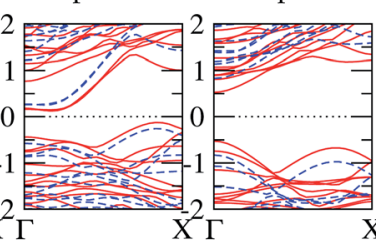

t-s/o-AsNR H-t-s/o-AsNR ac-s/o-AsNR H-ac-s/o-AsNR

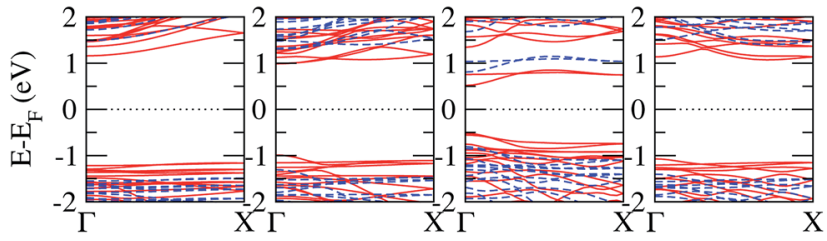

zz-b-AsNR H-zz-b-AsNR ac-b-AsNR H-ac-b-AsNR

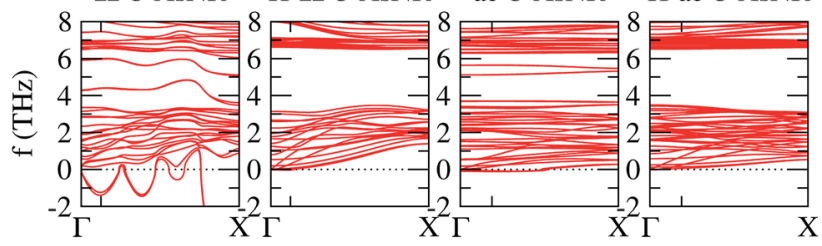

zz-p-AsNR H-zz-p-AsNR ac-p-AsNR H-ac-p-AsNR

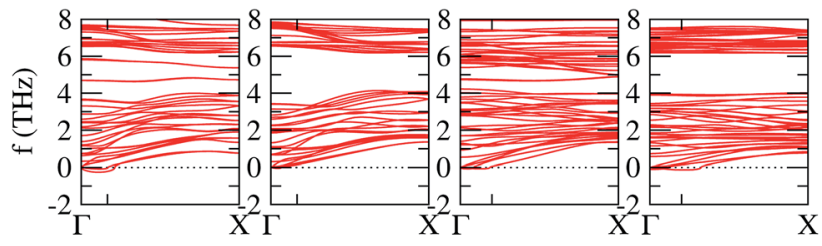

t-s/o-AsNR H-t-s/o-AsNR ac-s/o-AsNR H-ac-s/o-AsNR

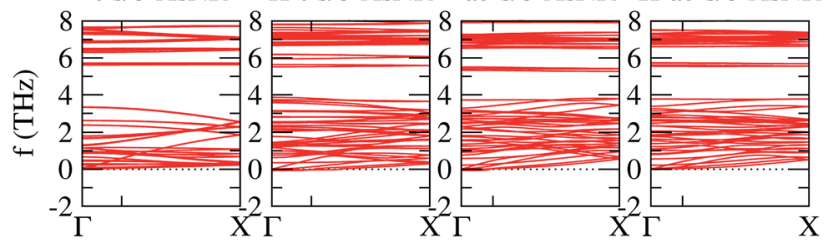

Fig. 5 Upper panels: energy band structures for the AsNRs shown in Fig. 1, as calculated with PBE (continuous red lines) and HSE (dashed blue lines); the Fermi energy, $E_{\mathrm{F}}$, is set to zero. Lower panels: lowfrequency region of the phonon band structures of the same AsNRs; the only tick on each horizontal axis signals the first reciprocal-space point commensurate with the supercell used in the DFT calculations.

band gaps than their pristine counterparts. On the other hand, recent PBE/GGA studies on zigzag and armchair buckled (or blue) phosphorene nanoribbons (zz-b-PNRs and ac-b-AsNRs) have shown that, in pristine form, ultra-narrow zz-b-PNRs are metallic, while ac-b-PNRs exhibit an oscillating behavior of the electronic band gap; after edge passivation with hydrogen atoms, the band gaps of both kinds of ribbons become large. ${ }^{29}$

A key question is now whether the AsNRs can possibly be synthesized. Mechanical stability is a necessary but nonsufficient requirement, which we assessed by checking if the phonon spectra of the AsNRs contain any imaginary frequencies. However, the use of periodic boundary conditions in DFT calculations leads to slight violations of the isotropy of free space that introduce artifacts in the phonon dispersions near the $\Gamma$ point.$^{30}$ Hence, small imaginary frequencies of the flexural branches between $\Gamma$ and the first reciprocal-space point commensurate with the supercell are not necessarily indicative of instability. We therefore allowed for imaginary frequencies with an absolute value up to $0.25 \mathrm{THz}$.

The phonon spectra of non-passivated AsNRs have a maximum frequency of around $8 \mathrm{THz}$, compatible with the value for the bulk $2 \mathrm{D}$ phases. ${ }^{14}$ Passivation with hydrogen introduces new vibrational modes; specifically, all H-AsNRs spectra studied have two narrow bands centered around 18 $\mathrm{THz}$ and $60 \mathrm{THz}$, originating from sets of very flat phonon bands. Analysis of the projected densities of vibrational states reveals that such modes are almost completely localized on the hydrogen atoms: the higher-frequency band involves stretching of the As- $\mathrm{H}$ bonds while the band around $18 \mathrm{THz}$ corresponds to rotations of the $\mathrm{H}$ atoms around their neighboring As. The localized character of those modes is due to the very large difference in mass between the two chemical species, and similar partitions of the phonon spectrum have been observed in other 2D systems containing hydrogen, like germanane. ${ }^{31}$ Since those phonon branches are clearly separated from the lowest-frequency bundle, we focus our attention on the ranges of frequencies below $8 \mathrm{THz}$ to enable easier comparisons between hydrogenated and non-hydrogenated ribbons. The lower panels of Fig. 5 show the phonon spectra of the nanoribbons depicted in Fig. 1 in this frequency range; following the usual convention, imaginary frequencies are plotted as negative values. All of these nanoribbons are mechanically stable except for the zz-b-AsNR, which is clearly unstable with respect to symmetric and antisymmetric longitudinal displacements of its edge atoms.

Some finer features of the phonon spectra of most mechanically stable AsNRs are also strongly reminiscent of their parent structures: all of them have a noticeable gap between two dense bundles of branches $(f \lesssim 4 \mathrm{THz}$ and $f \gtrsim 6 \mathrm{THz}$ ), with a narrow band inside that gap in the case of s/o-AsNRs just like in bulk s/o arsenene. ${ }^{14}$ Differences due to confinement and reconstruction are, however, important: e.g., as expected in any system with one-dimensional periodicity, the number of acoustic modes in the AsNRs is four instead of three, and two of them have parabolic dispersions for one in the bulk 2D phase. The spectra are generally less bulk-like (e.g. with a less clearly defined gap) in non-passivated AsNRs. This points to the relevance of the very reactive edges and the more extensive reconstruction in those cases. Perhaps the most unique among the spectra is that of the t-s/o-AsNR, with its particular reconstruction in weakly interacting nanowires and the corresponding sharp decrease in the number of nondegenerate phonon branches.

The next step in our assessment of stability, with a special focus on passivated $v s$. non-passivated comparisons, involves adding in the effect of atomic vibrations to the energetic analysis. As illustrated in ref. 14, this can change the conclusions 
even at $0 \mathrm{~K}$, and lead to crossovers at finite temperature. Let us consider two generic nanoribbons of types I and II. Each unit cell of type I (II) contains $n_{\mathrm{As}}^{\mathrm{I}}\left(n_{\mathrm{As}}^{\mathrm{II}}\right)$ arsenic atoms and $n_{\mathrm{H}}^{\mathrm{I}}\left(n_{\mathrm{H}}^{\mathrm{II}}\right)$ hydrogen atoms, so the following process is balanced:

$$
n_{\mathrm{As}}^{\mathrm{II}} \mathrm{AsNR}^{\mathrm{I}}+\frac{n_{\mathrm{As}}^{\mathrm{I}} n_{\mathrm{H}}^{\mathrm{II}}}{2} \mathrm{H}_{2} \Leftrightarrow n_{\mathrm{As}}^{\mathrm{I}} \mathrm{AsNR}^{\mathrm{II}}+\frac{n_{\mathrm{As}}^{\mathrm{II}} n_{\mathrm{H}}^{\mathrm{I}}}{2} \mathrm{H}_{2} .
$$

To study its free-energy balance, we took into account the translational and vibrational contributions for the $\mathrm{H}_{2}$ molecules in the gas phase (modeled as an ideal gas) and the vibrational contribution for the nanoribbons. Under this approximation, it is thermodynamically favorable for the process to evolve towards the side of the AsNR with the higher value of the following free energy per As atom:

$$
\begin{aligned}
\Delta F= & \frac{n_{\mathrm{H}}}{2 n_{\mathrm{As}}}\left\{E\left(\mathrm{H}_{2}\right)+F_{\mathrm{vib}}\left(\mathrm{H}_{2}\right)+k_{\mathrm{B}} T\left[\log \left(\frac{p}{n_{\mathrm{Q}} k_{\mathrm{B}} T}\right)-1\right]\right\} \\
& -\frac{1}{n_{\mathrm{As}}}\left[E(\mathrm{AsNR})+F_{\text {vib }}(\mathrm{AsNR})\right] .
\end{aligned}
$$

Here, $F_{\text {vib }}\left(\mathrm{H}_{2}\right)$ and $F_{\text {vib }}($ AsNR) are the vibrational contributions to the free energy of an $\mathrm{H}_{2}$ molecule and the AsNR, respectively, calculated using the same harmonic expressions as in ref. 14 . The pressure-related term accounts for the translational contribution to the free energy of a $\mathrm{H}_{2}$ molecule. A pressure of $p$ $=1 \mathrm{~atm}$ is assumed, and $n_{\mathrm{Q}}$ is calculated from the molecular mass $(m)$ of $\mathrm{H}_{2}$ as

$$
n_{\mathrm{Q}}=\left(\frac{m k_{\mathrm{B}} T}{2 \pi \hbar^{2}}\right)^{\frac{3}{2}}
$$

From the plots of that free energy, similar for all the ribbon widths considered (Fig. 6), it can be seen that the H-passivated and non-passivated ac-s/o-AsNRs are the least stable phases in their corresponding categories at any temperature. This is in dramatic contrast with the case of the bulk, where the s/o structure is the most stable at high temperatures, ${ }^{\mathbf{1 4}}$ and shows that the vibrational contribution to the free energy is far less decisive in nanoribbons. The most stable phase among non-
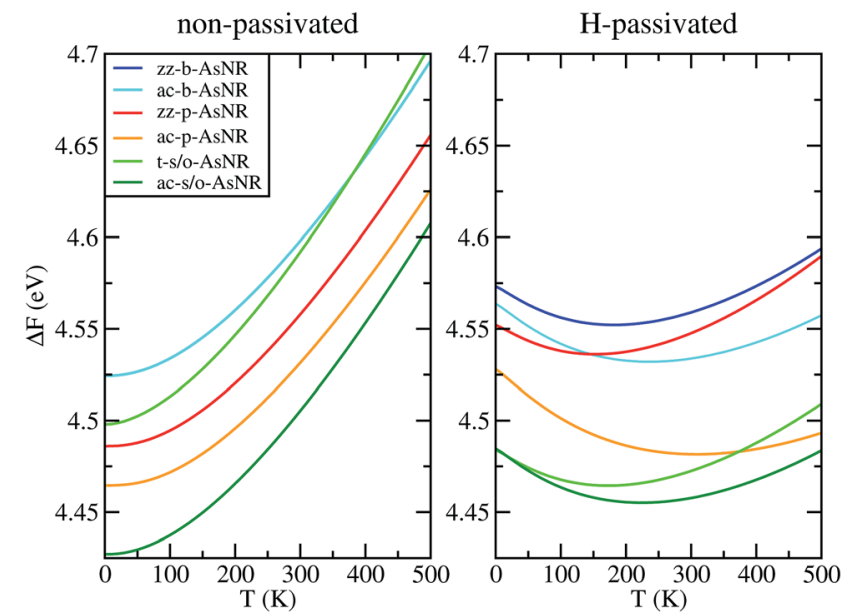

Fig. 6 Free energy per arsenic atom of the passivated and nonpassivated AsNRs shown in Fig. 1 as a function of temperature, as defined in the text. passivated AsNRs is the ac-b-AsNR except at very high temperature, where the t-s/o-AsNR becomes more favorable; in the $\mathrm{H}$ passivated case, the H-zz-b-AsNR dominates in the whole temperature range. Perhaps even more interesting is the comparison among all phases, with or without hydrogen. Edge passivation has a strong stabilizing effect at low temperatures, but even for the phases with higher $\Delta F$ this effect can be overcome by increasing $T$ due to the contribution from the highfrequency vibrational modes of the $\mathrm{H}$-passivated ribbons once they become significantly thermally activated. A notable exception to this general explanation is, once more, the t-s/oAsNR, which is more stable in the non-passivated version even at vanishing temperature thanks to its very special reconstruction.

\section{Conclusions}

To sum up, our results show that many of the AsNRs considered here are wide-bandgap semiconductors, i.e., have gaps in the 2$4 \mathrm{eV}$ range. Therefore, they are good candidates for the design of new semiconductor devices able to operate at much higher voltages, frequencies and temperatures than conventional semiconductors like Si. In particular, all t-s/o-AsNRs have band gaps around $3 \mathrm{eV}$, the highest among all the non-passivated ribbons; most ac-b-AsNRs have band gaps between 2 and $2.5 \mathrm{eV}$; and all $\mathrm{H}$-t-s/o-AsNRs, H-ac-b-AsNRs, H-zz-b-AsNRs and $\mathrm{H}$-ac-s/o-AsNRs, as well as the thinnest $\mathrm{H}$-zz-p-AsNRs and $\mathrm{H}$-acp-AsNRs, fall within the $2-4 \mathrm{eV}$ band gap range. Non-passivated ribbons (t-s/o-AsNRs and ac-b-AsNRs, also the most stable according to free-energy calculations) are more relevant at high $T$, since the stabilization due to edge passivation is overcome by thermal activation of vibrational modes involving the $\mathrm{H}$ atoms. Besides, when deposited on an inert substrate to build an electronic device, edge passivation by means of $\mathrm{H}$ is not commonly sought. In that regard, it is worth noting that the stability of these nanostructures can be further enhanced when grown on appropriate substrates. With an eye put on miniaturization and low-dimensionality, t-s/o-AsNRs deserve particular attention. Our results show that they can be seen as an array of weakly interacting nanowires, a fact that points to the possibility of reaching the thinnest stable nanoribbon so far.

\section{Conflicts of interest}

There are no conflicts to declare.

\section{Acknowledgements}

This work was supported by the Xunta de Galicia (AGRUP2015/ 11, ED431E 2018/8 and GRC ED431C 2016/001) and by the Junta de Castilla y León (Project No. VA124G18). The first two of these research projects were partially supported by FEDER.

\section{References}

1 Y. Zhang, A. Rubio and G. L. Lay, J. Phys. D: Appl. Phys., 2017, 50, 053004 . 
2 K. Takeda and K. Shiraishi, Phys. Rev. B: Condens. Matter Mater. Phys., 1994, 50, 14916-14922.

3 S. Cahangirov, M. Topsakal, E. Aktürk, H. Şahin and S. Ciraci, Phys. Rev. Lett., 2009, 102, 236804.

4 P. Vogt, P. De Padova, C. Quaresima, J. Avila, E. Frantzeskakis, M. C. Asensio, A. Resta, B. Ealet and G. Le Lay, Phys. Rev. Lett., 2012, 108, 155501.

5 M. E. Dávila, L. Xian, S. Cahangirov, A. Rubio and G. L. Lay, New J. Phys., 2014, 16, 095002.

6 M. Derivaz, D. Dentel, R. Stephan, M.-C. Hanf, A. Mehdaoui, P. Sonnet and C. Pirri, Nano Lett., 2015, 15, 2510-2516.

7 A. H. Castro Neto, F. Guinea, N. M. R. Peres, K. S. Novoselov and A. K. Geim, Rev. Mod. Phys., 2009, 81, 109-162.

8 J.-A. Yan, R. Stein, D. M. Schaefer, X.-Q. Wang and M. Y. Chou, Phys. Rev. B: Condens. Matter Mater. Phys, 2013, 88, 121403.

9 V. Georgakilas, M. Otyepka, A. B. Bourlinos, V. Chandra, N. Kim, K. C. Kemp, P. Hobza, R. Zboril and K. S. Kim, Chem. Rev., 2012, 112, 6156-6214.

10 L. Li, Y. Yu, G. J. Ye, Q. Ge, X. Ou, H. Wu, D. Feng, X. H. Chen and Y. Zhang, Nat. Nanotechnol., 2014, 9, 372-377.

11 H. Liu, A. T. Neal, Z. Zhu, Z. Luo, X. Xu, D. Tománek and P. D. Ye, ACS Nano, 2014, 8, 4033-4041.

12 Z. Zhu and D. Tománek, Phys. Rev. Lett., 2014, 112, 176802. 13 F. Ersan, E. Aktürk and S. Ciraci, Phys. Rev. B: Condens. Matter Mater. Phys., 2016, 94, 245417.

14 J. Carrete, L. J. Gallego and N. Mingo, J. Phys. Chem. Lett., 2017, 8, 1375-1380.

15 S. Zhang, Z. Yan, Y. Li, Z. Chen and H. Zeng, Angew. Chem., Int. Ed., 2015, 54, 3112-3115.

16 C. Kamal and M. Ezawa, Phys. Rev. B: Condens. Matter Mater. Phys., 2015, 91, 085423.
17 Y. Wang and Y. Ding, Nanoscale Res. Lett., 2015, 10, 254.

18 J. Zhang, H. J. Liu, L. Cheng, J. Wei, J. H. Liang, D. D. Fan, J. Shi, X. F. Tang and Q. J. Zhang, Sci. Rep., 2014, 4, 6452.

19 H.-S. Tsai, S.-W. Wang, C.-H. Hsiao, C.-W. Chen, H. Ouyang, Y.-L. Chueh, H.-C. Kuo and J.-H. Liang, Chem. Mater., 2016, 28, 425-429.

20 G. Kresse and J. Hafner, Phys. Rev. B: Condens. Matter Mater. Phys., 1993, 47, 558-561.

21 G. Kresse and J. Furthmüller, Phys. Rev. B: Condens. Matter Mater. Phys., 1996, 54, 11169-11186.

22 A. Togo and I. Tanaka, Scr. Mater., 2015, 108, 1-5.

23 J. P. Perdew, K. Burke and M. Ernzerhof, Phys. Rev. Lett., 1996, 77, 3865-3868.

24 J. Heyd, G. E. Scuseria and M. Ernzerhof, J. Chem. Phys., 2003, 118, 8207-8215.

25 J. Heyd, G. E. Scuseria and M. Ernzerhof, J. Chem. Phys., 2006, 124, 219906.

26 J. Paier, M. Marsman, K. Hummer, G. Kresse, I. C. Gerber and J. G. Ángyán, J. Chem. Phys., 2006, 124, 154709.

27 J. Paier, M. Marsman, K. Hummer, G. Kresse, I. C. Gerber and J. G. Ángyán, J. Chem. Phys., 2006, 125, 249901.

28 D. Kecik, E. Durgun and S. Ciraci, Phys. Rev. B: Condens. Matter Mater. Phys., 2016, 94, 205409.

29 R. Swaroop, P. K. Ahluwalia, K. Tankeshwar and A. Kumar, RSC Adv., 2017, 7, 2992-3002.

30 J. Carrete, W. Li, L. Lindsay, D. A. Broido, L. J. Gallego and N. Mingo, Mater. Res. Lett., 2016, 4, 204-211.

31 G. Coloyan, N. D. Cultrara, A. Katre, J. Carrete, M. Heine, E. Ou, J. Kim, S. Jiang, L. Lindsay, N. Mingo, D. Broido, J. P. Heremans, J. Goldberger and L. Shi, Appl. Phys. Lett., 2016, 109, 131907. 